\title{
Lotus essential oil improves neurite elongation and facilitates functional recovery after peripheral nerve injury
}

\author{
NAPATR SRIRAKSA ${ }^{1}$, RATCHANIPORN KONGSUI ${ }^{1,2}$ and SITTHISAK THONGRONG ${ }^{2,3}$ \\ ${ }^{1}$ Division of Physiology, School of Medical Sciences; \\ ${ }^{2}$ The Unit of Excellence in Translational Neurosciences Initiative, University of Phayao; \\ ${ }^{3}$ Division of Anatomy, School of Medical Sciences, University of Phayao, Amphur Muang, Phayao 56000, Thailand
}

Received December 8, 2021; Accepted February 8, 2022

DOI: $10.3892 b r .2022 .1513$

\begin{abstract}
Injury to the peripheral nerve may lead to deficits in nerve function. An increase in the levels of free radicals plays a role in inhibition of nerve regeneration following damage. The aim of this study was to investigate the effects of lotus essential oil (LEO) on neurite outgrowth in vitro and nerve regeneration in vivo in a rat model of sciatic nerve crush injury. Gas chromatography-mass spectrometry analysis showed that the principal constituent of LEO was palmitic acid ethyl ester $(25.12 \%)$. The radical scavenging activity of LEO was evaluated using the DPPH method, and was determined to be $\mathrm{IC}_{50}=29.01 \pm 2.93 \mu \mathrm{g} / \mathrm{ml}$. LEO-treated sensory neurons exhibited increased neurite outgrowth and upregulated levels of phospho-ERK. Sensory and motor functions were improved in rats treated with 50 and $100 \mathrm{mg} / \mathrm{kg} \mathrm{LEO}$, and this was accompanied by an increase in the number of neurons in the dorsal root ganglia, as well as an increase in the nerve axon diameters following nerve injury. Taken together, these results suggests that LEO may serve as a novel pharmacological option for the management of peripheral nerve injury.
\end{abstract}

\section{Introduction}

Peripheral nerve injury (PNI) is a serious chronic disease that can result in motor and sensory function impairments. Furthermore, PNI causes the death of motor neurons in the spinal cord and sensory neurons in dorsal root ganglia (DRG), which can result in reduced motor and sensory recovery $(1,2)$. PNI consequently induces axonal demyelination and degeneration, and this can result in the absence of reinnervation to the target organs leading to more severe morphological changes, such as muscular atrophy $(3,4)$. Therefore, faster

Correspondence to: Dr Sitthisak Thongrong, Division of Anatomy, School of Medical Sciences, University of Phayao, 19 Moo 2 Tambon Maeka, Amphur Muang, Phayao 56000, Thailand E-mail: sitthisak.th@up.ac.th

Key words: Nelumbo nucifera, lotus essential oil, peripheral nerve injury, functional recovery, axon elongation long-distance regeneration of nerves is required for improvement of functional recovery. Following PNI, oxidative stress is increased at the site of the injured nerve and this plays a crucial role in exacerbating damage and hindering nerve reformation (5). Free radical molecules, such as reactive oxygen species, destroy numerous molecules in the cell, resulting in mitochondrial dysfunction, lipid peroxidation and eventually cellular apoptosis (6).

Nelumbo nucifera ( $N$. nucifera), also known as lotus or Bualuang in Thai, is an aquatic plant in the family Nelumbonaceae, and it is widely cultivated in Asian countries (7). All the parts of $N$. nucifera are used in traditional herbal medicines and have been reported to exhibit several beneficial pharmacological effects, including antimicrobial, anticancer and anti-inflammatory activity $(8,9)$. Extracts of $N$. nucifera seeds can increase superoxide dismutase and catalase levels, which results in increased free radical scavenging activity in rat kidneys and liver (10). A study in a diabetic rat model showed that extracts from lotus leaves could decrease diabetes-induced nephropathy (11). Aporphine alkaloids extracted from $N$. nucifera flowers were shown to promote neurite outgrowth in PC-12 cells (12). In addition, it has been shown that the major component in lotus flower oil is palmitic acid, which possesses antioxidant activity and can increase the phosphorylation of ERK and can increase cellular proliferation $(13,14)$.

Since oxidative stress is increased following PNI, and free radicals play a role in inhibiting axonal regeneration and increasing cellular apoptosis following nerve injury, the aim of the present study was to investigate the effects of lotus flower oil on neurite outgrowth and nerve regeneration in an in vivo model of PNI.

\section{Materials and methods}

Gas chromatography-mass spectrometry analysis of lotus flower oil. Whole lotus flowers were used for extraction using an absolute extraction method from Tropicalife Co., Ltd., according to the manufacturer's protocol. The organic compounds of the lotus essential oil (LEO) were analyzed using GC-MS by Scientific and Technological Instruments Center of Mae Fah Luang University, Thailand. The operating conditions were as follows: The capillary column (HP-5 ms) 
was used with a flow rate of $1 \mathrm{ml} / \mathrm{min}$. The gas chromatograph oven was set at $40-240^{\circ} \mathrm{C}$. Hexane was used as the solvent. The temperature of the injector and detector were set at 250 and $230^{\circ} \mathrm{C}$, respectively. The mass spectrometer was set to determine a molecular weight range of 20-300 Dalton. The organic compounds were identified by comparing their retention times and mass spectra with the data from the National Institute of Standards and Technology Mass Spectra database (NIST08). The relative quantity of each compound was presented by area $\%$.

Antioxidant activity of LEO using the DPPH method. LEO antioxidant activity was evaluated by assessing the radical scavenging effect of the stable DPPH free radical. According to the method described by Kumaran et al (15), $50 \mu \mathrm{l} \mathrm{LEO}$ at various concentrations $(5,10,20,40,80,160,320$ and $640 \mu \mathrm{g} / \mathrm{ml})$ was added to $200 \mu 10.1 \mathrm{mM}$ DPPH-ethanol solution in a 96-well plate. After $30 \mathrm{~min}$ of incubation in the dark at $25^{\circ} \mathrm{C}$, the absorbance was determined at $520 \mathrm{~nm}$ using a Cytation5 microplate reader (BioTek Instruments, Inc.) and the DPPH radical scavenging activity was calculated as the $\mathrm{IC}_{50}$ using the formula: $\%$ inhibition $=\left[\left(\mathrm{A}_{\text {control }}-\mathrm{A}_{\text {sample }}\right) / \mathrm{A}_{\text {control }}\right] \mathrm{x} 100$; where $\mathrm{A}_{\text {control }}$ is the absorbance of DPPH without sample and $\mathrm{A}_{\text {sample }}$ is the absorbance of DPPH with LEO. The experiment was performed three times for each sample.

DRG cell culture and treatment. The dilution of essential oil in ethanol was performed as described by Shinomiya et al (16) was used. Briefly, LEO was diluted $50 \%(\mathrm{v} / \mathrm{v})$ in $70 \%$ ethanol before treatment. The final concentration of ethanol was $0.01 \%$ in RPMI medium. DRG cell culture was performed as described by Hausott et al (17). Briefly, male Wistar rats were sacrificed using $\mathrm{CO}_{2}$ at a volume displacement rate of $30-70 \%$ of the chamber per min for $5 \mathrm{~min}$.

Death was confirmed by cardiac arrest, the spinal cord was exposed and $\sim 45$ DRGs were collected in cold RPMI medium (Invitrogen; Thermo Fisher Scientific, Inc.). Thereafter, DRGs were incubated in collagenase type I $(5,000 \mathrm{U} / \mathrm{ml}$, Invitrogen; Thermo Fisher Scientific, Inc.) for $1 \mathrm{~h}$ at $37^{\circ} \mathrm{C}$ followed by $0.25 \%$ trypsin/EDTA (Invitrogen; Thermo Fisher Scientific, Inc.) for $15 \mathrm{~min}$ at $37^{\circ} \mathrm{C}$. DRGs were next washed 3 times in RPMI medium containing $10 \%$ horse serum and $5 \%$ fetal bovine serum (Sigma-Aldrich; Merck KGaA), and then transferred to culture medium (RPMI supplemented with B27; Invitrogen; Thermo Fisher Scientific, Inc.) and dissociated using fire-polished Pasteur pipettes. The cell suspension was seeded onto plastic dishes coated with poly-D-lysine hydrobromide (100 $\mu \mathrm{g} / \mathrm{ml}$, Sigma-Aldrich; Merck KGaA) at $37^{\circ} \mathrm{C}$ overnight and then coated with laminin $(5 \mu \mathrm{g} / \mathrm{ml}$; Sigma-Aldrich ; Merck $\mathrm{KGaA}$ ) at $37^{\circ} \mathrm{C}$ for $4 \mathrm{~h}$. A total of $1 \mathrm{~h}$ after seeding the medium was changed and the cells were treated with $0.15,0.3$ or $0.6 \mu \mathrm{l} / \mathrm{ml}$ LEO in RPMI medium supplemented with B27 supplement and an antibiotic-antimycotic (Invitrogen; Thermo Fisher Scientific, Inc.). Cells were incubated in a humidified incubator at $37^{\circ} \mathrm{C}$ with $5 \% \mathrm{CO}_{2}$ for $24 \mathrm{~h}$. The DRG neuron cultures were performed three times.

Sciatic nerve crush model. Male Wistar rats with an initial weight of 200-220 g (6-8 weeks old) were obtained from Nomura Siam International Co., Ltd. The rats were housed in a temperature $\left(25 \pm 2^{\circ} \mathrm{C}\right)$ and humidity $(35-60 \%)$ controlled room with a $12 \mathrm{~h}$ light/dark cycle, and provided ad libitum access to food and water. The rats were randomly divided into a control, sham, sciatic nerve injury (SNI), SNI $+50 \mathrm{mg} / \mathrm{kg}$ LEO or SNI + $100 \mathrm{mg} / \mathrm{kg}$ LEO group (6 rats/group). To induce SNI, the rats were anesthetized using $50 \mathrm{mg} / \mathrm{kg}$ intraperitoneal injection of sodium pentobarbital. The nerve crush procedure was performed on the left hind limb. An incision was made in the middle of the thigh, and the muscles were carefully incised at the intermuscular septum without cutting the muscle fibers to expose the sciatic nerve. The nerve was clamped at $1 \mathrm{~cm}$ proximal to the bifurcation using artery forceps (straight $12 \mathrm{~cm}$ for $30 \mathrm{sec}$. The sham rats underwent the same surgery without clamping of the nerve. Thereafter, the skin was sutured with a nylon suture to close the wound without stitching up muscles to avoid muscle damage $(18,19)$. After surgery, all rats were an intraperitoneal injection of $10 \mathrm{mg} / \mathrm{kg}$ meloxicam to provide pain relief and reduce inflammation for 3 consecutive days.

Foot withdrawal test. Thermal stimulation was applied to determine recovery of sensory function on days 7, 14, 21 and 28 after surgery (20). Prior to the test, the rats were placed on a non-heated plate for $10 \mathrm{~min}$. Subsequently, the paw withdrawal latency $(\mathrm{PWL})$ in response to a heat stimulus $\left(50^{\circ} \mathrm{C}\right)$ using a hot plate at the plantar side of the hind paw was recorded. The rats were not allowed contact with the heated plate for more than $20 \mathrm{sec}$. PWL was recorded on the left paw three times and the time interval between tests was $5 \mathrm{~min}$. The experiment was repeated three times with each rat.

Walking track analysis. The recovery of motor function was assessed on days 7, 14, 21 and 28 after surgery using walking tract analysis. Briefly, the hind paws of trained rats were pressed down onto a stamp pad soaked with water-soluble black ink. The rats were allowed to walk along a confined box $(30 \times 90 \times 20 \mathrm{~cm})$, which was placed on a white paper $(21 \times 90 \mathrm{~cm})$. The footprints were recorded to calculate the sciatic functional index (SFI) as described by Bain et al (21). The experiment was repeated three times with each rat.

Immunocytochemistry and histological analysis. After $24 \mathrm{~h}$, DRG neurons were fixed with $4 \%$ paraformaldehyde at $25^{\circ} \mathrm{C}$ for $30 \mathrm{~min}$. Cells were washed three times with PBS and permeabilized with $0.5 \%$ Triton X-100 (Sigma-Aldrich; Merck KGaA) at $25^{\circ} \mathrm{C}$ for $5 \mathrm{~min}$, and blocked with $10 \%$ goat serum (Thermo Fisher Scientific, Inc.) in PBS at $25^{\circ} \mathrm{C}$ for $30 \mathrm{~min}$. Cells were incubated with primary antibodies against neuron specific $\beta$-III tubulin (Thermo Fisher Scientific, Inc.; cat. no. MA1-118; 1:1,000) and phospho- (p-)ERK (Cell Signaling Technology, Inc.; cat. no. $3179 ; 1: 250)$ at $4^{\circ} \mathrm{C}$ overnight. Secondary antibodies (Alexa-594 chicken anti-mouse; cat. no. A-21201) for $\beta$-III tubulin staining and Alexa-488 goat anti-rabbit (cat. no. A32731) for p-ERK staining; both at 1:1,000; Invitrogen; Thermo Fisher Scientific, Inc.) were applied for $30 \mathrm{~min}$ at $25^{\circ} \mathrm{C}$. Immunofluorescence labeled neurons were observed using an inverted fluorescence microscope (Zeiss Axiovert 100, Carl Zeiss AG; magnification, x20 objective lens). p-ERK fluorescence intensity measurements and neurite outgrowth were determined using Metamorph ${ }^{\odot}$ version 7. Evaluation of the average fluorescence intensity was performed after background 
subtraction. The longest neurite from the cell body to the growth cone was measured and taken as the maximal distance (22).

Histological analysis was performed on day 28 after nerve injury. The rats were sacrificed using $\mathrm{CO}_{2}$ (30-70\% volume displacement rate for $5 \mathrm{~min}$ ), the sciatic nerve and L4-L6 DRG were fixed in $4 \% \mathrm{PFA}$ at $4^{\circ} \mathrm{C}$ for $48 \mathrm{~h}$ followed by $15 \%$ sucrose in PBS for $24 \mathrm{~h}$ and then $30 \%$ sucrose in PBS for $48 \mathrm{~h}$. Sections were cut (20 $\mu \mathrm{m}$ thick) using a cryostat (Leica Microsystems, Inc., CM1950) for hematoxylin \& eosin (H\&E) (Sigma-Aldrich; Merck KGaA) staining. The sections were stained for $10 \mathrm{~min}$ with hematoxylin followed by $20 \mathrm{sec}$ with eosin both at $25^{\circ} \mathrm{C}$. DRG neurons were counted in area of $80,000 \mu \mathrm{m}^{2}$ using a $40 \mathrm{x}$ objective lens, and axon diameters were measured under a 100x oil immersion lens. Tissue morphology was determined using the ECLIPSE Ni-U I Upright Microscopes (Nikon Corporation) and analyzed using NIS Elements imaging software version 5 (Nikon Corporation). All histological experiments were performed three times.

Statistical analysis. Data are presented as the mean \pm SD for DPPH analysis or SEM for DRG cell culture, tissue morphological analysis and behavioral tests. A one-way ANOVA followed by a Tukey's post-hoc test was used to analyze the data in GraphPad Prism version 9 (GraphPad Software, Inc.). P<0.05 was considered to indicate a statistically significant difference.

\section{Results}

Composition analysis and assessment of the free radical scavenging activity of LEO. There were three primary compounds that individually accounted for $>10 \%$ of the total content of LEO: Palmitic acid ethyl ester (25.12\%), linoleic acid ethyl ester (18.17\%) and methyl 8,11,14-heptadecatrienoate $(10.45 \%)$ (Table I). DPPH is widely used to evaluate radical scavenging activity (23-25). Ascorbic acid and Trolox (both from Sigma-Aldrich; Merck KGaA) were used as positive controls antioxidants. LEO had ability to inhibit DPPH radical activity with an $\mathrm{IC}_{50}$ value of $29.01 \pm 2.93 \mu \mathrm{g} / \mathrm{ml}$ (Table II).

LEO promotes neurite elongation and ERK phosphorylation. DRG neurons were cultured and treated with various concentration of LEO. The length of the longest neurite of DRG neurons was measured as the maximal distance to indicate the effect of LEO on axon elongation. Neurite outgrowth was observed after $24 \mathrm{~h}$ of treatment, LEO at concentrations of 0.3 and $0.6 \mu \mathrm{l} / \mathrm{ml}$ showed a significant increase in maximal distance when compared with the negative control and vehicle control groups (both $\mathrm{P}<0.001$, Fig. 1). LEO-mediated promotion of neurite outgrowth may be mediated via ERK activation. Elevation of the p-ERK/ERK ratio plays a crucial role in axonal elongation in vivo (22). Phosphorylation of ERK was investigated by immunofluorescence staining of DRG neurons after $24 \mathrm{~h}$ of LEO treatment. Average fluorescence intensity of the p-ERK/ERK ratio in DRG neurons treated with 0.3 or $0.6 \mu \mathrm{l} / \mathrm{ml}$ LEO was significantly higher compared with the negative control and vehicle control groups $(\mathrm{P}<0.01$ and $\mathrm{P}<0.001$, respectively; Fig. 2).

Histological analysis of DRG and sciatic nerve after crush injury. To study the effect of LEO on PNI, sciatic nerve and
Table I. Gas chromatography-mass spectrometry analysis of lotus essential oil.

\begin{tabular}{lcr}
\hline Compound name & $\begin{array}{c}\text { Retention } \\
\text { time, min }\end{array}$ & Area, \% \\
\hline (6Z,9E)-Heptadeca-6,9-diene & 29.46 & 2.62 \\
Palmitic acid methyl ester & 34.66 & 2.79 \\
Palmitic acid ethyl ester & 35.40 & 25.12 \\
Heneicosane & 36.28 & 7.26 \\
Linoleic acid ethyl ester & 36.93 & 18.17 \\
Methyl 8,11,14-heptadecatrienoate & 37.07 & 10.45 \\
Methyl 2-methylhexadecanoate & 37.23 & 7.09 \\
Hexadecane & 38.12 & 2.78 \\
\hline
\end{tabular}

Table II. DPPH radical inhibition by lotus essential oil.

\begin{tabular}{lc}
\hline Test samples & $\mathrm{IC}_{50} \pm \mathrm{SD}, \mu \mathrm{g} / \mathrm{ml}$ \\
\hline Lotus essential oil & $29.01 \pm 2.93$ \\
Ascorbic acid & $1.37 \pm 0.21$ \\
Trolox & $2.89 \pm 0.54$ \\
\hline
\end{tabular}

L4-L6 DRG were cut and stained with H\&E. After 28 days of SNI, injured rats orally administered 50 or $100 \mathrm{mg} / \mathrm{kg} \mathrm{LEO}$ showed a significant increase in the number of DRG neurons compared with the SNI group $(\mathrm{P}<0.001$ and $\mathrm{P}<0.05$, respectively; Fig. 3). Axon diameters were measured to assess the effect of LEO on nerve regeneration. On day 28 after SNI, axon diameters were decreased in all injured animals. However, oral administration of 50 or $100 \mathrm{mg} / \mathrm{kg}$ LEO significantly increased axon diameter when compared to the SNI group ( $\mathrm{P}<0.05$, Fig. 4).

LEO promotes functional recovery after SNI. The in vitro and in vivo data indicated that LEO enhanced neurite elongation, and promoted DRG neuron survival and nerve regeneration. Therefore, whether oral administration of LEO exerted a therapeutic effect on sensory and motor function recovery was next assessed in vivo. Prior to establishment of the SNI model, all animals were tested behaviorally to obtain baseline values of locomotor function using walking tract analysis and sensory function using a thermo-withdrawal test. The baseline values did not differ between animals. After SNI, injured animals exhibited sensory and motor function deficits. Behaviors were assessed over a 4-week period. Sensory function recovery was improved in mice treated with 50 or $100 \mathrm{mg} / \mathrm{kg}$ LEO by day 21 after SNI $(\mathrm{P}<0.05)$. The differences became more pronounced by day $28(\mathrm{P}<0.001)$ (Fig. 5A).

Motor function recovery was assessed by walking tract analysis. The SFI gradually improved with time. Significant differences between SNI alone vs. SNI + 50 or $100 \mathrm{mg} / \mathrm{kg}$ LEO were observed on week 4 after lesion $(\mathrm{P}<0.01$ and $\mathrm{P}<0.001$, respectively; Fig. 5B). Overall, the results indicated that administration of LEO improved sensory and motor functions in a rat model of SNI, and this corresponded with enhanced nerve regeneration. 


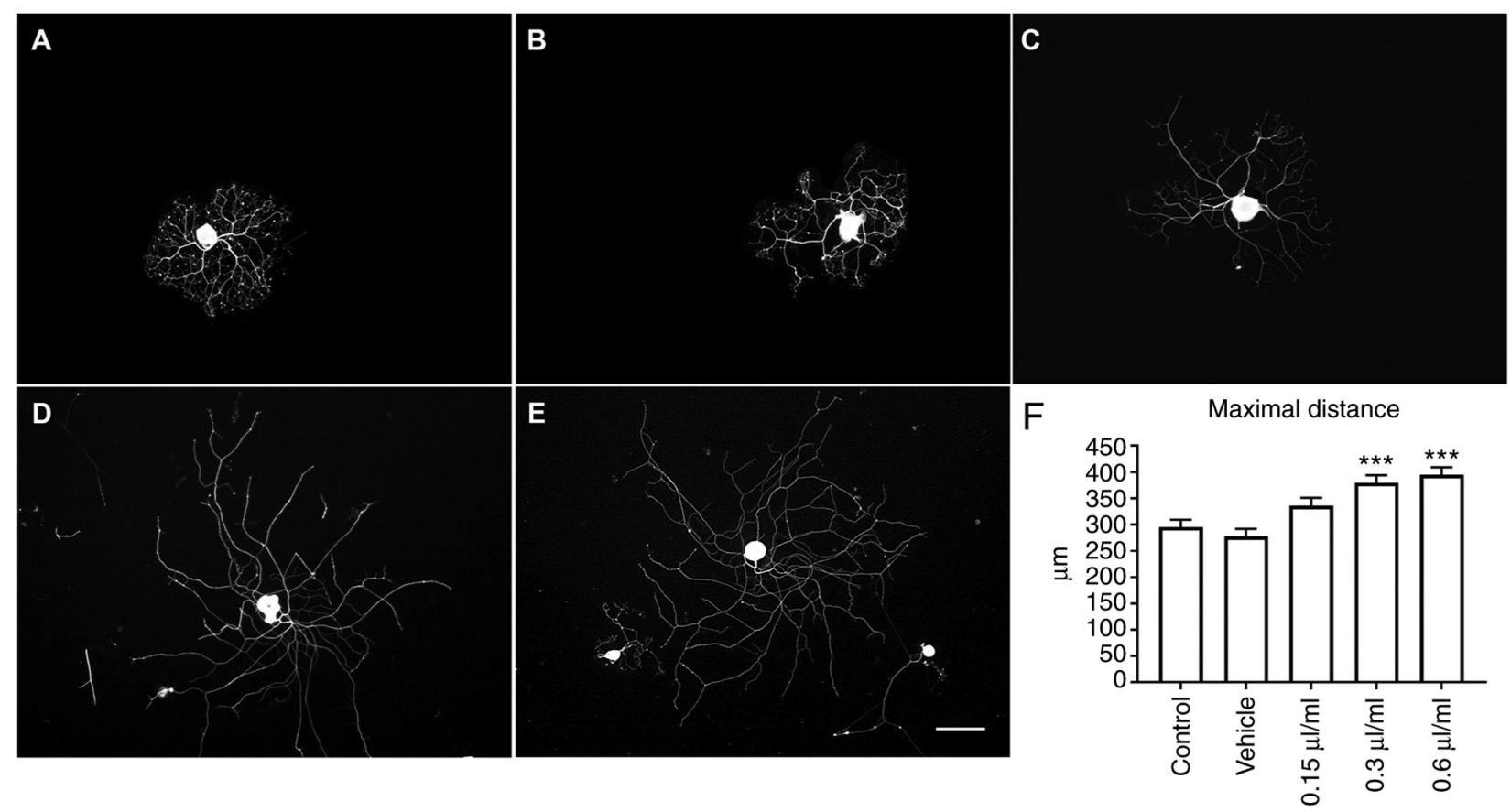

Figure 1. Effect of LEO on neurite outgrowth, DRG neurons were treated with various concentrations of LEO for $24 \mathrm{~h}$. DRG neurons treated with 0.3 and $0.6 \mu 1 / \mathrm{ml}$ LEO exhibited increased neurite elongation when compared with the control and vehicle groups. (A) Control, (B) vehicle, (C) $0.15 \mu 1 / \mathrm{ml} \mathrm{LEO}$, (D) $0.3 \mu 1 / \mathrm{ml}$ LEO and (E) $0.6 \mu \mathrm{l} / \mathrm{ml} \mathrm{LEO.} \mathrm{(F)} \mathrm{Quantitative} \mathrm{analysis} \mathrm{of} \mathrm{neurite} \mathrm{length.} \mathrm{Data} \mathrm{are} \mathrm{presented} \mathrm{as} \mathrm{the} \mathrm{mean} \pm$ SEM of three independent experiments. Scale bar, $100 \mu \mathrm{m}$. ${ }^{* * *} \mathrm{P}<0.001$ vs. control and vehicle groups. LEO, lotus essential oil; DRG, dorsal root ganglion.

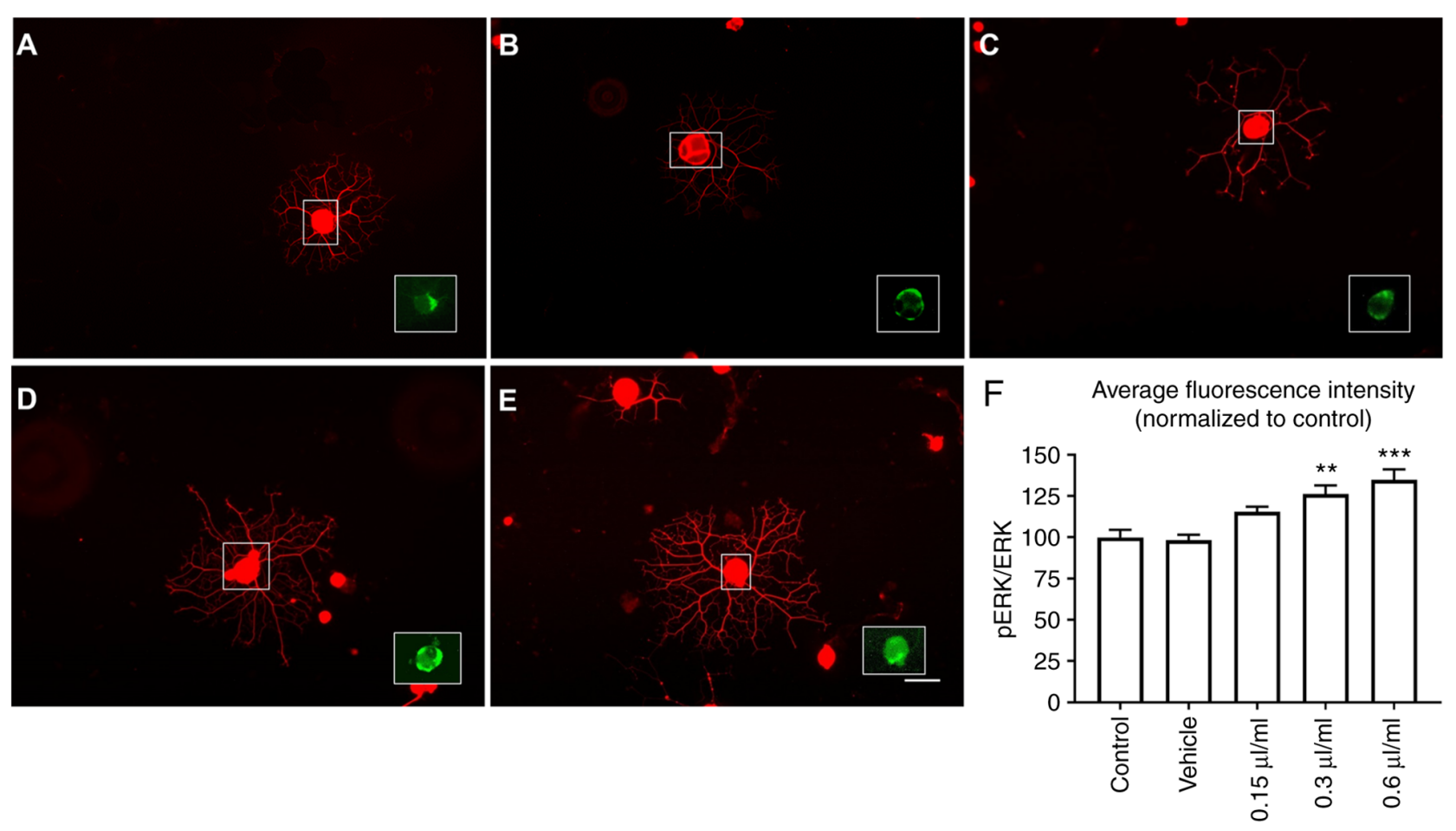

Figure 2. Effect of LEO on ERK activation, DRG neurons were treated with LEO for $24 \mathrm{~h}$. When treated with 0.3 or $0.6 \mu 1 / \mathrm{ml}$, DRG neurons exhibited a significant increase in the ratio of p-ERK/ERK fluorescence. (A) Control, (B) vehicle, (C) $0.15 \mu 1 / \mathrm{ml} \mathrm{LEO,} \mathrm{(D)} 0.3 \mu 1 / \mathrm{ml} \mathrm{LEO}$ and (E) $0.6 \mu 1 / \mathrm{ml} \mathrm{LEO}$. (F) Quantitative analysis of p-ERK/ERK fluorescence intensity. Data are presented as the mean \pm SEM of three independent experiments. Scale bar, $100 \mu \mathrm{m}$. ${ }^{* *} \mathrm{P}<0.01,{ }^{* * *} \mathrm{P}<0.001$ vs. control and vehicle groups. LEO, lotus essential oil; DRG, dorsal root ganglion; $\mathrm{p}-$, phospho-.

\section{Discussion}

Local upregulation of free radicals is observed following PNI (26). Increases in the levels of free radical molecules has been shown to attenuate recovery of nerve function after injury (27). The levels of free radical molecules have been reported to increase after injury to the DRG and the sciatic nerve (28). The increase in the free radical levels also plays 

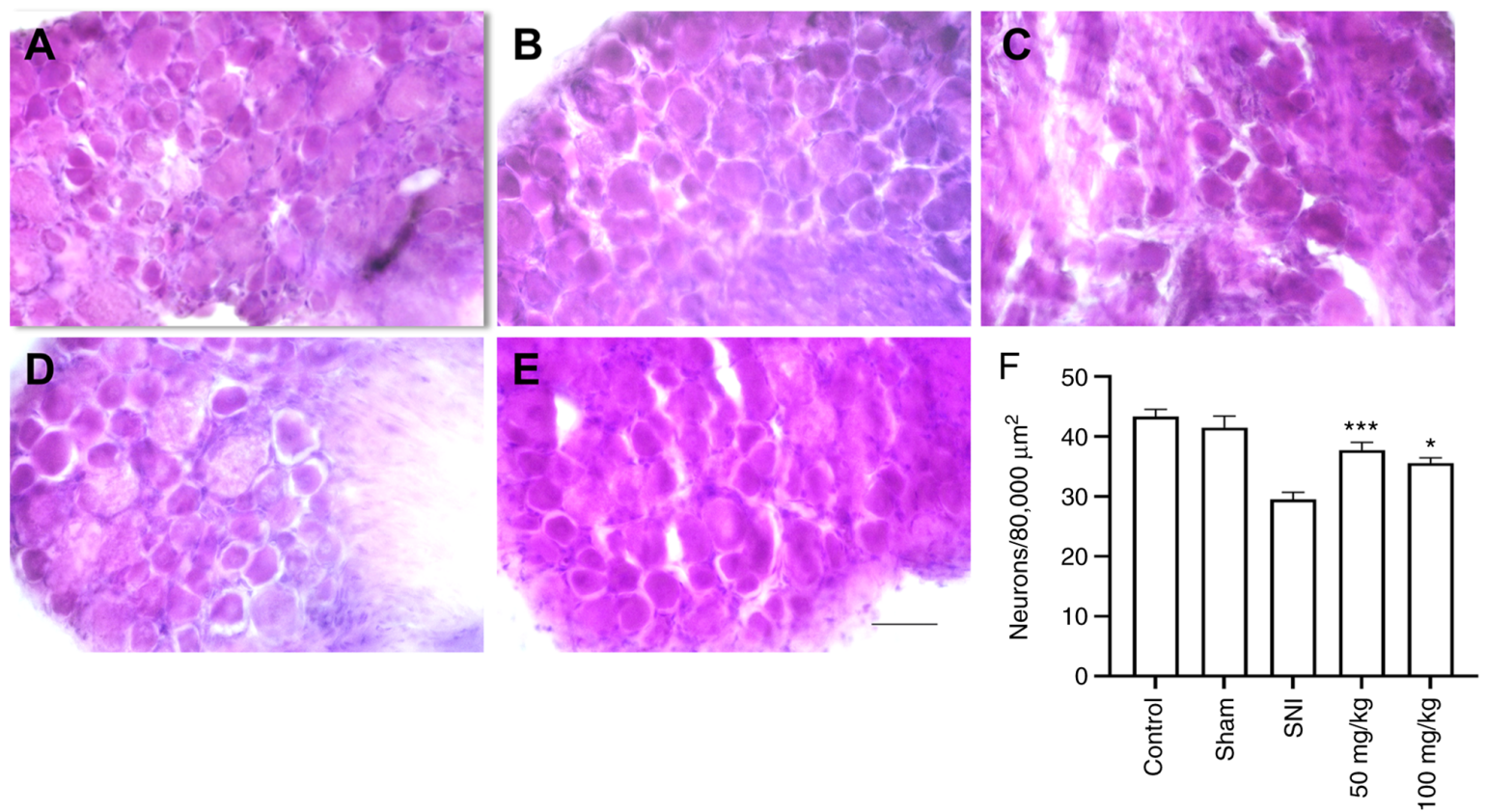

Figure 3. Effect of LEO on the survival of sensory neurons 28 days after SNI. Treatment with $50 \mathrm{or} 100 \mathrm{mg} / \mathrm{kg}$ LEO significantly increased the number of sensory neurons in the DRG compared with the SNI group. (A) Control, (B) sham, (C) SNI, (D) $50 \mathrm{mg} / \mathrm{kg} \mathrm{LEO} \mathrm{and} \mathrm{(E)} 100 \mathrm{mg} / \mathrm{kg}$. (F) Quantitative analysis of sensory neurons in DRG. Data are presented as the mean \pm SEM of three independent experiments. ${ }^{*} \mathrm{P}<0.05,{ }^{* * *} \mathrm{P}<0.001$ vs. SNI. Scale bar, $50 \mu \mathrm{m}$. LEO, lotus essential oil; SNI, sciatic nerve injury.
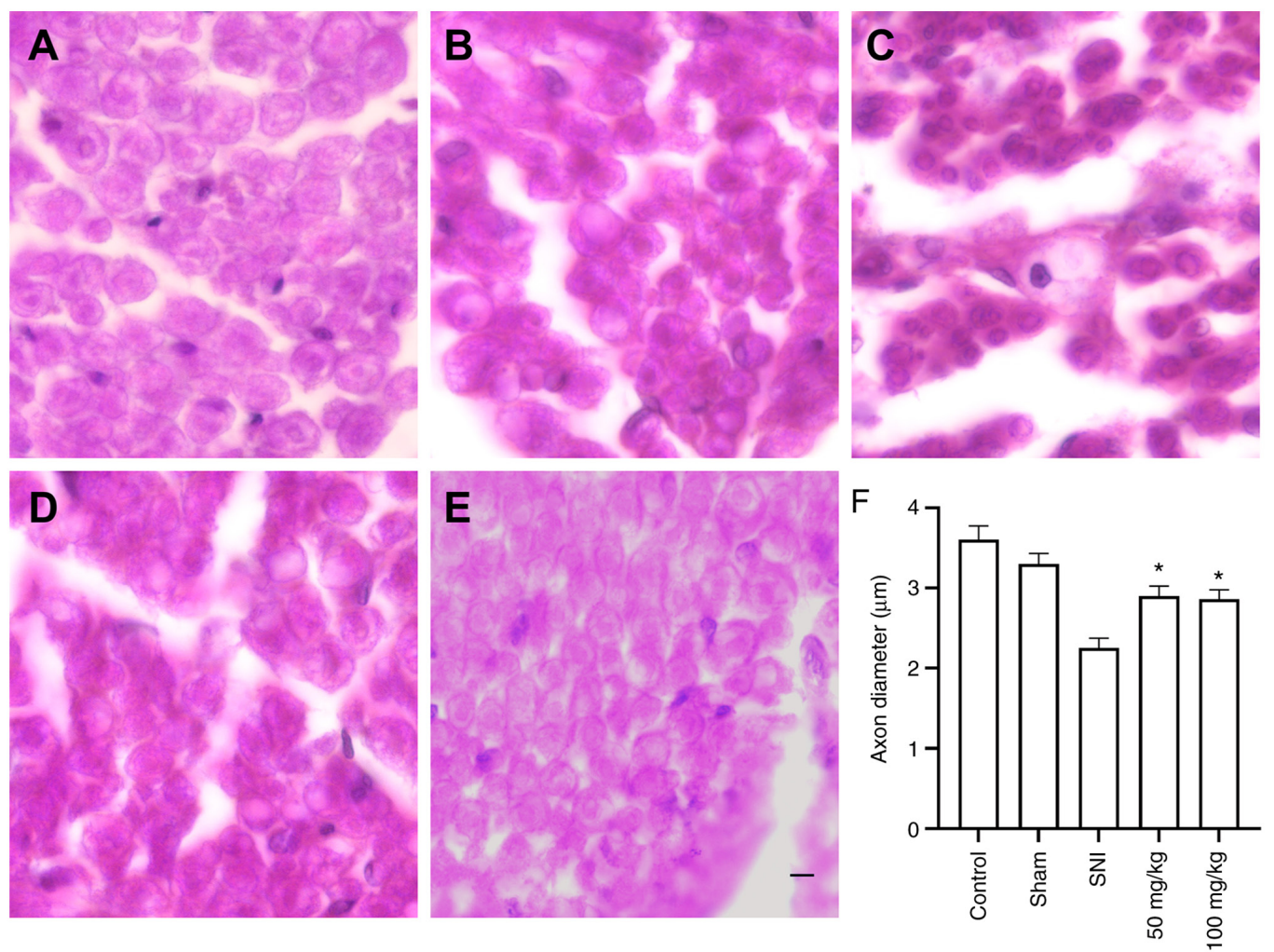

Figure 4. Histological analysis of nerve axon diameters. (A) Hematoxylin and eosin staining to assess the effect of LEO on axon diameter. The diameters of regenerated axons were significantly larger when treated with 50 or $100 \mathrm{mg} / \mathrm{kg}$ LEO compared with the SNI group. (A) Control, (B) sham, (C) SNI, (D) $50 \mathrm{mg} / \mathrm{kg}$ LEO and (E) $100 \mathrm{mg} / \mathrm{kg}$ LEO. (F) Quantitative analysis of nerve axon diameters. Data are presented as the mean \pm SEM of three independent experiments. ${ }^{*}<0.05$ vs. SNI. Scale bar, $5 \mu \mathrm{m}$. LEO, lotus essential oil; SNI, sciatic nerve injury.

a role in inducing nerve degeneration and interferes with the regeneration process of the injured nerve (29). In this study, the effect of LEO, the crude extract from lotus flower, on nerve functional recovery after injury was assessed. The results from the GC-MS analysis showed three major components of lotus flower oil, including palmitic acid, linoleic acid and methyl 

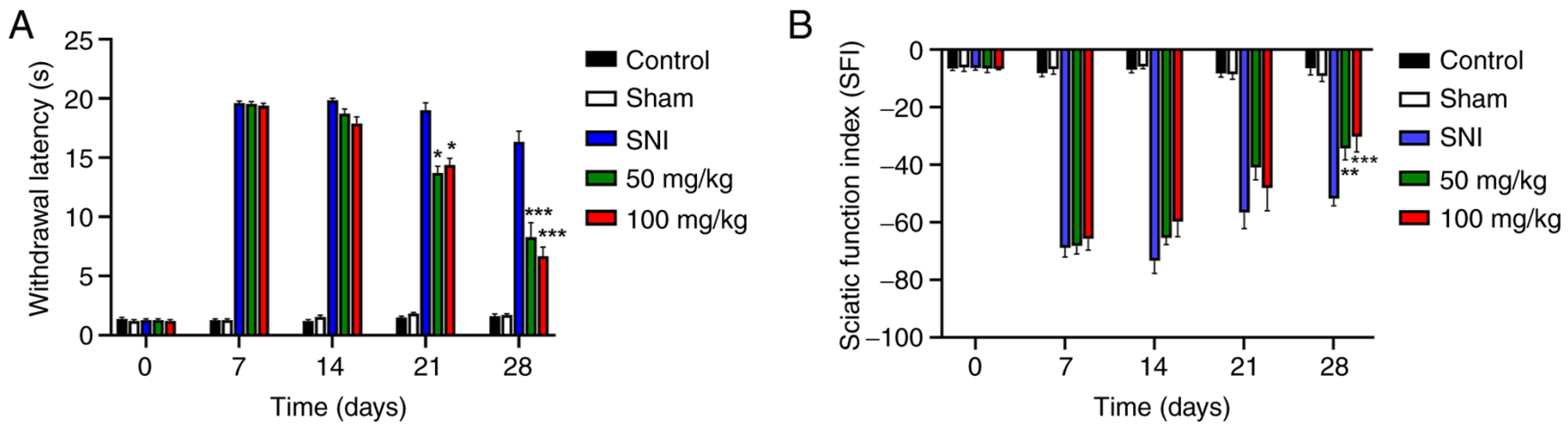

Figure 5. LEO promotes functional recovery after SNI. (A) Foot withdrawal latency throughout the experiment was used to assess sensory recovery as shown. (B) Motor function recovery was assessed using walking track analysis and presented as the sciatic function index on days $7,14,21$ and 28 after $\mathrm{SNI}$. ${ }^{*} \mathrm{P}<0.05$, ${ }^{* *} \mathrm{P}<0.01,{ }^{* * *} \mathrm{P}<0.001$ vs. SNI group. Data are presented as the mean \pm SEM. $\mathrm{n}=6$ rats. LEO, lotus essential oil; SNI, sciatic nerve injury.

8,11,14-heptadecatrienoate. A previous study reported that palmitic acid is a fatty acid that exhibits antioxidant activity (30). To confirm whether LEO exhibited radical scavenging activity, the DPPH method was used, and the results showed that LEO exhibited potent DPPH radical scavenging activity with an $\mathrm{IC}_{50}$ value of $29.01 \pm 2.93 \mu \mathrm{g} / \mathrm{ml}$. A previous study reported that the antioxidant and DPPH radical scavenging activities of lotus extract could prevent oxidative stress-induced neuronal death in the central nervous system (15). Faster axon elongation and nerve re-innervation are required in nerve recovery after injury (31). The results of the present study showed that, treatment of DRG neurons with LEO resulted in increased axonal distance growth compared with the control and vehicle-treated groups. LEO induced neurite outgrowth and elongation in vitro, and this was likely associated with the upregulation of ERK activation, as shown through increased ERK phosphorylation. Stimulation of ERK activation has been reported to promote neurite outgrowth in vitro (32) and axon regrowth after nerve transection (33); thus, these are important processes for nerve regeneration. Lu et al (34) reported that the accumulation of antioxidant molecules at the site of the injured nerve resulted in an increase in the ratio of $\mathrm{p}-\mathrm{ERK} / \mathrm{ERK}$, and this could improve axon regeneration.

A notable finding of the present study was that LEO enhanced neurite outgrowth and elongation, both of which are essential processes for nerve repair and functional recovery. Thus the effect of LEO on functional recovery of nerves was assessed in the sciatic nerve crush model. After sciatic nerve injury, the animals exhibited motor and sensory neuron death as a result of impaired nerve regeneration (35). A previous study reported that the recovery of nerve sensory function following PNI could be defined by the number of cells that survived within the DRG (36). A decrease in DRG neurons after PNI can reduce sensory functional recovery (37). The results of the present study showed that administration of LEO could prevent DRG neurons from death after sciatic nerve lesion. Animals treated with 50 and $100 \mathrm{mg} / \mathrm{kg}$ LEO showed an increase in the number of DRG neurons compared with the animals in the control group. A previous study showed that antioxidants from the lotus plant could protect neurons from death following acute nerve injury (38). The neuroprotective effect of LEO on DRG neurons was likely associated with the antioxidant effect and increased phosphorylation of ERK activation (34). The rescue of sensory function was evaluated using thermal stimulation. Rats with sciatic nerve lesion exhibited an increased foot withdrawal threshold. By day 21 of LEO treatment, sensory function was improved in the animals treated with LEO compared with the untreated SNI animals. Of note, the improvement in sensory function continually increased with the treatment period. This result also correlates with the higher numbers of neurons in the DRG.

In the present study, it was shown that treatment with LEO could improve locomotor functional recovery by day 28 after sciatic nerve crush. Axon regrowth and remyelination are required for reinnervation of target tissues, and these are associated with functional motor recovery (39). Remyelination after injury to the peripheral nerve is possible and required to connect the nerve to the skin or other target tissues (40). The results of the present study showed that the axon diameters were increased in the LEO treated groups compared with the SNI group. Since free radicals and oxidative stress have been reported to damage the peripheral nerve and delay its functional recovery (41), increased axon diameters in LEO treated groups may be the result of the antioxidant activity of lotus flower oil. Upregulation of antioxidant enzymes, such as glutathione reductase, can increase the axon diameter and density after SNI. Therefore, antioxidative systems are also involved in the recovery process after nerve lesions (42). Thus, the ability of LEO to increase radical scavenging activity, neurite elongation activity and functional recovery of the nerve may have been achieved through the synergistic action of different bioactive components. However, the effects of the primary components of LEO individually have not been tested on neurite elongation and functional nerve recovery, therefore, this is a limitation of the present study and the subject of a future study. Further experiments are required to determine the individual effects of the constituents of LEO separately in the PNI model. LEO is a rich source of several long-chain fatty acids; however, the effect of these fatty acids on functional nerve recovery have not been assessed. Therefore, additional experiments are to study the effects of these long-chain fatty acids, such as stearic acid, oleic acid and linolenic acid on PNI. Antioxidants have been shown to promote nerve recovery after injury. Ascorbyl palmitate, a fat-soluble form of ascorbic acid with potent antioxidant activity, should be assessed future experiments of sciatic nerve injury. Moreover, LEO can promote nerve regeneration and 
functional recovery after crush injury, and this may be due to the effects of LEO on the expression of proteins. Thus, the expression levels of proteins related to remyelination, such as myelin protein zero and peripheral myelin protein 2 , should be assessed to better understand the effect of LEO on the mechanism of nerve remyelination.

In summary, injury to the peripheral nerve results in motor and sensory functional impairment, and administration of LEO accelerated functional nerve recovery. Lotus, a plant that possesses antioxidant activity, can prevent sensory neurons from death and promote an increase in axon diameter. Based on the results of the present study, LEO may be a potential beneficial therapeutic option for the management of PNI. However, the effect of LEO on the activity and levels of endogenous antioxidant enzymes remains unclear. Therefore, additional studies are required to investigate the effect of LEO on endogenous antioxidant enzyme activities after nerve injury.

\section{Acknowledgements}

Not applicable.

\section{Funding}

This study was supported in part by funding from the School of Medical Sciences, University of Phayao (Phayao, Thailand; grant no. MS 201017).

\section{Availability of data and materials}

The datasets used and/or analyzed during the present study are available from the corresponding author on reasonable request.

\section{Authors' contributions}

ST and NS wrote the manuscript, analyzed the data and designed the study. RK collected the data. All authors have read and approved the final manuscript. ST, NS and RK confirm the authenticity of all the raw data.

\section{Ethics approval and consent to participate}

All animal experiments were approved by the Animal Ethics Committee of University of Phayao (Phayao, Thailand; approval no. 630104008).

\section{Patient consent for publication}

Not applicable.

\section{Competing interests}

The authors declare that there are no competing interests.

\section{References}

1. Kinugasa T, Ozaki S, Hamanaka S and Kudo N: The effects of sciatic nerve axotomy on spinal motoneurons in neonatal Bax-deficient mice. Neurosci Res 44: 439-446, 2002.
2. Shi TJ, Hua XY, Lu X, Malkmus S, Kinney J, Holmberg K, Wirz S, Ceccatelli S, Yaksh T, Bartfai T and Hökfelt T: Sensory neuronal phenotype in galanin receptor 2 knockout mice: Focus on dorsal root ganglion neurone development and pain behaviour. Eur J Neurosci 23: 627-636, 2006.

3. Zachodne DL and Ho T: Endoneural microenvironment and acute nerve crush injury in the rat sciatic nerve. Brain Res 535: 43-48, 1990.

4. Rasul A, Al-shawi AA, Malik SA, Anwar H, Rasool B, Razzaq A, Aziz N, Kamran SKS, Sarfraz I, Shabbir A, et al: Neurada procumbens promotes functions regain in a mouse model of mechanically induced sciatic nerve injury. Pak J Pharm Sci 32 (Suppl 4): S1761-S1766, 2019.

5. Dong L, Li R, Li D, Wang B, Lu Y, Li P, Yu F, Jin Y, Ni X, Wu Y, et al: FGF10 enhances peripheral nerve regeneration via the preactivation of the PI3K/Akt signaling-mediated antioxidant response. Front Pharmacol 16: 1224, 2019.

6. Wang $\mathrm{CH}, \mathrm{Wu} \mathrm{SB}, \mathrm{Wu} \mathrm{YT}$ and Wei YH: Oxidative stressresponse elicited by mitochondrial dysfunction: Implication in thepathophysiology of aging. Exp Biol Med (Maywood) 238: 450-460, 2013.

7. Ono Y, Hattori E, Fukaya Y, Imai S and Ohizumi Y: Anti-obesity effect of Nelumbo nucifera leaves extract in mice and rats. J Ethnopharmacol 106: 238-244, 2006.

8. Park E, Kim GD, Go MS, Kwon D, Jung IK, Auh JH and Kim JH: Anti-inflammatory effects of Nelumbo leaf extracts and identification of their metabolites. Nutr Res Pract 11: 265-274, 2017.

9. Liu CP, Tsai WJ, Lin YL, Liao JF, Chen CF and Kuo YC: The extracts from Nelumbo nucifera suppress cell cycle progression, cytokine genes expression, and cell proliferation in human peripheral blood mononuclear cells. Life Sci 75: 699-716, 2004.

10. Rai S, Wahile A, Mukherjee K, Saha BP and Mukherjee PK: Antioxidant activity of Nelumbo nucifera (sacred lotus) seeds. J Ethnopharmacol 104: 322-327, 2006.

11. Chen HW, Yang MY, Hung TW, Chang YC and Wang CJ: Nelumbo nucifera leaves extract attenuate the pathological progression of diabetic nephropathy in high-fat diet-fed and streptozotocin-induced diabetic rats. J Food Drug Anal 27: 736-748, 2019.

12. Yano M, Nakashima S, Oda Y, Nakamura S and Matsuda H: BBB-permeable aporphine-type alkaloids in Nelumbo nucifera flowers with accelerative effects on neurite outgrowth in PC-12 cells. J Nat Med 74: 212-218, 2020

13. Jeon S, Kim NH, Koo BS, Kim JY and Lee AY: Lotus (Nelumbo nuficera) flower essential oil increased melanogenesis in normal human melanocytes. Exp Mol Med 41: 517-525, 2009.

14. Schevzov G, Kee AJ, Wang B, Sequeira VB, Hook J, Coombes JD, Lucas CA, Stehn JR, Musgrove EA, Cretu A, et al: Regulation of cell proliferation by ERK and signal-dependent nuclear translocation of ERK is dependent on Tm5NM1-containing actin filaments. Mol Biol Cell 26: 2475-2490, 2015.

15. Kumaran A, Ho CC and Hwang LS: Protective effect of Nelumbo nucifera extracts on beta amyloid protein induced apoptosis in PC12 cells, in vitro model of Alzheimer's disease. J Food Drug Anal 26: 172-181, 2018.

16. Shinomiya M, Kawamura K, Tanida E, Nagoshi M, Motoda H, Kasanami Y, Hiragami $F$ and Kano Y: Neurite outgrowth of PC12 mutant cells induced by orange oil and d-limonene via the p38 MAPK pathway. Acta Med Okayama 66: 111-118, 2012.

17. Hausott B, Vallant N, Auer M, Yang L, Dai F, Brand-Saberi B and Klimaschewski L: Sprouty2 down-regulation promotes axon growth by adult sensory neurons. Mol Cell Neurosci 42: 328-340, 2009.

18. Ramli D, Aziz I, Mohamad M, Abdulahi D and Sanusi J: The changes in rats with sciatic nerve crush injury supplemented with evening primrose oil: Behavioural, morphologic, and morphometric analysis. Evid Based Complement Alternat Med 2017: 3476407, 2017.

19. Sriraksa N, Kongsui R, Thongrong S, Duangjai A and Hawiset T: Effect of Azadirachta Indica flower extract on functional recovery of sciatic nerve crush injury in rat models of DM. Exp Ther Med 17: 541-550, 2019.

20. Menéndez L, Lastra A, Hidalgo A and Baamonde A: Unilateral hot plate test: A simple and sensitive method for detecting central and peripheral hyperalgesia in mice. J Neurosci Methods 113: 91-97, 2002.

21. Bain JR, Mackinnon SE and Hunter DA: Functional evaluation of complete sciatic, peroneal, and posterior tibial nerve lesions in the rat. Plast Reconstr Surg 83: 129-138, 1989. 
22. Marvaldi L, Thongrong S, Kozłowska A, Irschick R, Pritz CO, Bäumer B, Ronchi G, Geuna S, Hausott B and Klimaschewski L: Enhanced axon outgrowth and improved long-distance axon regeneration in sprouty2 deficient mice. Dev Neurobiol 75: 217-231, 2015.

23. Yang D, Wang Q, Ke L, Jiang J and Ying T: Antioxidant activities of various extracts of lotus (Nelumbo nuficera Gaertn) rhizome. Asia Pac J Clin Nutr 16 (Suppl 1): S158-S163, 2007.

24. Gangwar M, Gautam MK, Sharma AK, Tripathi YB, Goel RK and Nath G: Antioxidant capacity and radical scavenging effect of polyphenol rich Mallotus philippenensis fruit extract on human erythrocytes: An in vitro study. ScientificWorldJournal 2014: 279451, 2014.

25. Huang B, Ban X, He J, Tong J, Tian J and Wang YL: Comparative analysis of essential oil components and antioxidant activity of extracts of Nelumbo nucifera from various areas of China J Agric Food Chem 58: 441-448, 2010.

26. Varija D, Kumar KP, Reddy KP and Reddy VK: Prolonged constriction of sciatic nerve affecting oxidative stressors \& antioxidant enzymes in rat. Indian J Med Res 129: 587-592, 2009.

27. Zhang L, Johnson D and Johnson JA: Deletion of Nrf2 impairs functional recovery, reduces clearance of myelin debris and decreases axonal remyelination after peripheral nerve injury. Neurobiol Dis 54: 329-338, 2013

28. Saray A, Apan A and Kisa U: Free radical-induced damage in experimental peripheral nerve injection injury. J Reconstr Microsurg 19: 401-406, 2003.

29. Hervera A, De Virgiliis F, Palmisano I, Zhou L, Tantardini E, Kong G, Hutson T, Danzi MC, Perry RB, Santos CXC, et al: Reactive oxygen species regulate axonal regeneration through the release of exosomal NADPH oxidase 2 complexes into injured axons. Nat Cell Biol 20: 307-319, 2018.

30. Tyagi T and Mala A: Phytochemical and GC-MS analysis of bioactive constituents in the ethanolic of Pistia stratiotes L. and Eichhornia crassipes (Mart.) solms. J Pharmacogn Phytochem 6: 195-206, 2017.

31. Jungnickel J, Haase K, Konitzer J, Timmer M and Grothe C: Faster nerve regeneration after sciatic nerve injury in mice over-expressing basic fibroblast growth factor. J Neurobiol 66 : 940-948, 2006

32. Wang X, Wang Z, Yao Y, Li J, Zhang X, Li C, Cheng Y, Ding G Liu L and Ding Z: Essential role of ERK activation in neurite outgrowth induced by $\alpha$-lipoic acid. Biochim Biophys Acta 1813: $827-838,2011$
33. Chierzi S, Ratto GM, Verma P and Fawcett JW: The ability of axons to regenerate their growth cones depends on axonal type and age, and is regulated by calcium, cAMP and ERK. Eur J Neurosci 21: 2051-2062, 2005.

34. Lu Y, Li R, Zhu J, Wu Y, Li D, Dong L, Li Y, Wen X, Yu F, Zhang H, et al: Fibroblast growth factor 21 facilitates peripheral nerve regeneration through suppressing oxidative damage and autophagic cell death. J Cell Mol Med 23: 497-511, 2019.

35. Kemp SW, Chiang CD, Liu EH, Wood MD, Willand MP, Gordon $\mathrm{T}$ and Borschel GH: Characterization of neuronal death and functional deficits following nerve injury during the early postnatal developmental period in rats. Dev Neurosci 37: 66-77, 2015.

36. Hart AM, Terenghi G and Wiberg M: Neuronal death after peripheral nerve injury and experimental strategies for neuroprotection. Neurol Res 30: 999-1011, 2008

37. McKay Hart A, Brannstrom T, Wiberg M and Terenghi G: Primary sensory neurons and satellite cells after peripheral axotomy in the adult rat: Timecourse of cell death and elimination. Exp Brain Res 142: 308-318, 2002.

38. Xiong W, MacColl Garfinkel AE, Li Y, Benowitz LI and Cepko CL: NRF2 promotes neuronal survival in neurodegeneration and acute nerve damage. J Clin Invest 125: 1433-1445, 2015.

39. Chen P, Piao X and Bonaldo P: Role of macrophages in Wallerian degeneration and axonal regeneration after peripheral nerve injury. Acta Neuropathol 130: 605-618, 2015.

40. Fatemi MJ, Foroutan KS, Ashtiani AK, Mansoori MJ, Vaghardoost R, Pedram S, Hosseinpolli A, Rajabi F and Mousavi SJ: Comparison of divided sciatic nerve growth within dermis, venous and nerve graft conduit in rat. J Res Med Sci 15: 208-213, 2010.

41. Levy D, Kubes P and Zochodne DW: Delayed peripheral nerve degeneration, regeneration, and pain in mice lacking inducible nitric oxide synthase. J Neuropathol Exp Neurol 60: 411-421, 2001.

42. Renno WM, Benov L and Khan KM: Possible role of antioxidative capacity of (-)-epigallocatechin-3-gallate treatment in morphological and neurobehavioral recovery after sciatic nerve crush injury. J Neurosurg Spine 27: 593-613, 2017.

This work is licensed under a Creative Commons Attribution-NonCommercial-NoDerivatives 4.0 International (CC BY-NC-ND 4.0) License. 